Proceedings

\title{
Thermophilic Actinobacteria Derived from Algerian Ecosystem as an Alternative Strategy for Salt Tolerance in Triticum durum ${ }^{+}$
}

\author{
Karima Loucif ${ }^{1,2}$, A Boulahrouf ${ }^{1}$ and $P$ Duez ${ }^{2}$ \\ 1 Laboratory of Microbiological Engineering and Applications, University of Brothers Mentouri, Constantine \\ 1, Chaâbat Erssas Campus, Ain El Bey Road, Constantine 25000, Algeria; \\ boulahroufabderrahmane@yahoo.fr \\ 2 Unit of therapeutic Chemistry and Pharmacognosy, Faculty of Medicine and Pharmacy, University of \\ Mons (UMONS), 7000 Mons, Belgium; pierre.duez@umons.ac.be \\ * Correspondence: karima25000@hotmail.com or karimaloucif25000@gmail.com; Tel.: +32-0485221570 or \\ +213-0659903244 \\ + Presented at the 1st International Electronic Conference on Plant Science, 1-15 December 2020; Available \\ online: https://iecps2020.sciforum.net/.
}

Published: 1 December 2020

\begin{abstract}
Durum wheat (Triticum durum Desf.) productivity is severely affected by major biotic and abiotic factors including salinity and phytopathogens fungi. Several approaches were used to reduce salinity effects on durum wheat, which included the use of Plant Growth Promoting Actinobacteria (PGPA). The present study was conducted to evaluate the ability of thermophilic actinobacteria, isolated from saharan sites to promote Durum wheat (Triticum durum Desf.) growth in the absence and presence of salt stress and to investigate their antifungal potential against Aspergillus niger. The effect of actinomycete inoculations in saline condition on Triticum durum behavior was studied. The salt stress was generated by application of different $\mathrm{NaCl}$ doses. The Screening for antifungal activity of actinomycetes isolates was done against phytopathogenic filamentous fungi Aspergillus niger by the agar cylinders method. Results demonstrated that thermophilic actinomycete strains promoted Durum wheat plant growth significantly even in the presence of salt. Inoculations with PGPR improved the plant growth, and increased the root and shoot lenghts, chlorophyll contents, and prolin content as compared to plants with no bacterial treatment (control). The PGPB strains WCD, OTH 1.3.1 and OTH 2.2.2.3 showed good in vitro antifungal (inhibition $>55 \%$ ) control.
\end{abstract}

Keywords: stress; salt; actinobacteria; thermophilic; phytopathogenic; Triticum durum

\section{Introduction}

Plants face various biotic and abiotic stresses in adverse environmental conditions. Among these, salinity is a major abiotic factor that suppresses agricultural productivity. For agriculture, saline stress is the most serious threat because salt deposition on arable land turns cultivatable land into unproductive area. Almost 3.6 billion hectares (Mha) of agriculture land (5.2 billion ha) is affected from soil degradation, erosion and salinity [1]. Plant-growth-promoting-bacteria (PGPB) are a group of microorganisms that enhance plant growth. PGPB promote plant growth by a higher acquisition of nutrients, increased availability of phytohormones, and a greater uptake of phosphorus and nitrogen.

The objectives of the present study were: (1) to determine the effect of six thermophilic actinobacteria isolated from saharan ecosystem of Southeast Algeria on roots and shoots elongation, 
proline accumulation and chlorophyll $\mathrm{a}$, chlorophyll $\mathrm{b}$ and chlorophyll tot under salinity stress; (2) to investigate their antifungal potential against Aspergillus niger by estimation of Percentage Growth Inhibition of filamentous fungi.

\section{Experiments}

\subsection{Actinomycete Strains}

Six actinomycete strains isolated from two different saharan sites in the Southeast region of Algeria (Biskra and Ouargla) as previously reported in (2.2), were investigated for their their salt tolerance capacity and their biocontrol activity against Aspergilllus niger.

\subsection{Screening for Antifungal Activity of Actinomycetes Isolates}

Six actinomycetes strains isolated from saharian soils and water of south east Algeria (OTH 1.2.2, OTH 1.3.1, OTH 2.2.2.3, WGS9, WCD, WSC2) were screened for their in vitro antifungal activity against phytopathogenic filamentous fungi Aspergillus niger provided by National Institute of Agronomic Research (N.I.A.R), El Khroub, Constantine. Algeria. by the agar cylinders method. The activity was tested in Sabouraud medium [peptone, $10 \mathrm{~g} \mathrm{l}^{-1}$, dextrose, $20 \mathrm{~g} \mathrm{l}^{-1}$, agar (Merck), $15 \mathrm{~g} \mathrm{l}^{-1}$, $\mathrm{pH}, 7$ ]. Actinobacteria isolates were grown on Bennett [glucose, $10 \mathrm{~g} \mathrm{l}^{-1}$, casaminoacid, $2 \mathrm{~g} \mathrm{l}^{-1}$, yeast extract, $1 \mathrm{~g} \mathrm{l}^{-1}$, beef extract, $1 \mathrm{~g} \mathrm{l}^{-1}$, agar (Merck), $15 \mathrm{~g} \mathrm{l}^{-1}, \mathrm{pH}, 7,3$ ] ; GLM [yeast extract, $3 \mathrm{~g} \mathrm{l}^{-1}$, malt extract, $3 \mathrm{~g} \mathrm{l}^{-1}$, peptone, $5 \mathrm{~g} \mathrm{l}^{-1}$, glucose, $10 \mathrm{~g} \mathrm{l}^{-1}$, agar (Merck), $\left.20 \mathrm{~g} \mathrm{l}^{-1}, \mathrm{pH}, 7,2\right]$; PELG [peptone, $5 \mathrm{~g} \mathrm{l}^{-}$ 1, Glucose, $\mathrm{g} \mathrm{l}^{-1}$, yeast extract, $2 \mathrm{~g} \mathrm{l}^{-1}$, agar (Merck), $\left.20 \mathrm{~g} \mathrm{l}^{-1}, \mathrm{pH}, 6,8-7\right]$ agar plates for 7 days at $28^{\circ} \mathrm{C}$.

Fungal mycelial cylinders ( $6 \mathrm{~mm}$ in diameter) prepared from the culture of the test germ was placed in the center of Sabouraud plates then actinobacteria agar cylinders (6 $\mathrm{mm}$ in diameter) where then taken and placed on the surface of the Sabouraud media. A plate with each target pathogen was used as the control. Plates were kept at $4{ }^{\circ} \mathrm{C}$ during $4 \mathrm{~h}$ for a good diffusion of the antifungal metabolite, then incubated at $25{ }^{\circ} \mathrm{C}$ for 48 hours and bioactivity was evaluated by measuring the diameter of inhibition zones (in $\mathrm{mm}$ ) around the cylinders agar and the percentage of fungal growth inhibition (GI) [3-6].

The percentage of fungal growth inhibition (GI) were calculated separately according to the following formula

\section{The inhibition zone $=\mathrm{C}-\mathrm{T}$ \\ Percentage Growth Inhibition $=[C-T / C] \times 100 \%$}

Where $\mathbf{C}$ and $\mathbf{T}$ were the diameters of fungal mycelial growth in the control and treated plates, respectively.

\subsection{Effect of the Actinomycete Strains on Seed Germination of Durum Wheat under Saline Stress}

\subsubsection{Greenhouse Pot Culture}

The six actinomycete strains were inoculated on Durum wheat seeds (Triticum durum Defs) of the Cirta variety. The seed surface was sterilized with a sodium hypochlorite solution $20 \%(v / v)$ and subsequently rinsed several times with sterile distilled water [7]. The inoculation was carried out by dipping the seeds in the different spore suspensions $\left(10^{6}\right.$ spores. $\left.\mathrm{mL}^{-1}\right)$ for germination; seeds immersed in sterile distilled water were used as a control Inoculated and uninoculated seeds were placed in pots $(\Phi=8 \mathrm{~cm})$ filled with soil (3 seeds/pot) and were irrigated with tap water twice a week [7]. After 7 days, of acclimatization with PGPR inoculation, $\mathrm{NaCl}$ solutions with different concentration ( $0 \mathrm{M}, 0,3 \mathrm{M}, 0,6 \mathrm{M}, 0,9 \mathrm{M}, 1,1 \mathrm{M}, 1,4 \mathrm{M}, 1,7 \mathrm{M})$ were added to one set and the other was kept without salt. Both inoculated and uninoculated treatments were replicated three times.

\subsubsection{Plant Analysis}


Forty days after sowing (DAS), plants were sampled, divided into shoots and roots separately. The following parameters were investigated: Lengths, free proline content and chlorophyll contents. Proline content of the leaves was determined by homogenizing the samples in a mortar and pestle in $3 \%$ sulfosalicylic acid. After centrifugation at $4500 \mathrm{rpm}$ for $45 \mathrm{~min}, 2 \mathrm{~mL}$ supernatant was added to 2 $\mathrm{mL}$ glacial acetic acid and $2 \mathrm{~mL}$ ninhydrin solution $(0.2 \mathrm{M})$. The mixture was kept at $95{ }^{\circ} \mathrm{C}$ for $60 \mathrm{~min}$, then, the reaction was stopped quickly by an ice bath. Toluene $(3 \mathrm{~mL})$ was added to the mixture and the organic phase was extracted and monitored at $520 \mathrm{~nm}$ by a spectrophotometer (Shimadzu, UV160) [8] Chlorophyll a (Chla), chlorophyll b (Chlb), and total chlorophyll (Chltot) contents were determined following the method described by Arnon [9]. $500 \mathrm{mg}$ of leaves from each sample, finely cut, were homogenized in $10 \mathrm{~mL}$ of acetone $80 \%$ and stored at $-10{ }^{\circ} \mathrm{C}$ overnight; after centrifugation at $3000 \times \mathrm{g}$ for $5 \mathrm{~min}$, supernatant absorbance was read at 663 for chlorophyll a and at $645 \mathrm{~nm}$ for chlorophyll $\mathrm{b}$. The chlorophyll concentrations in the samples were calculated through the following equations:

$$
\begin{gathered}
\mathrm{Chl}_{\mathrm{a}}\left(\mathrm{mg} \mathrm{L}^{-1}\right)=12.41(\mathrm{OD} 663)-2.59(\mathrm{OD} 645) \\
\mathrm{Chl}_{\mathrm{b}}\left(\mathrm{mg} \mathrm{L}^{-1}\right)=22.9(\mathrm{OD} 645)-4.68(\mathrm{OD} 663) \\
\mathrm{Chl}_{\text {tot }}\left(\mathrm{mg} \mathrm{L}^{-1}\right)=\mathrm{Chl}_{\mathrm{a}}+\mathrm{Chl}_{\mathrm{b}}
\end{gathered}
$$

\subsection{Statistical Analysis}

All data were presented as mean values of three replicates (mean \pm standard deviation). The data were analyzed using a statistical package, XLSTAT 2014 software (Addinsoft, Paris, France). Oneway ANOVA (analysis of variance) was employed utilizing Tukey's Honestly Significant Difference (HSD) post-hoc test to compare to determine the significant difference among means of the treatment at the level of $5 \%(p<0.05)$.

\section{Results}

\subsection{Antifungal Activity Detection}

All the actinomycetes strains present antifungal activity against Aspergillus niger. The highest percentage of the active strains is observed on the actinomycetes coming from the PELG medium (Figure 1a).

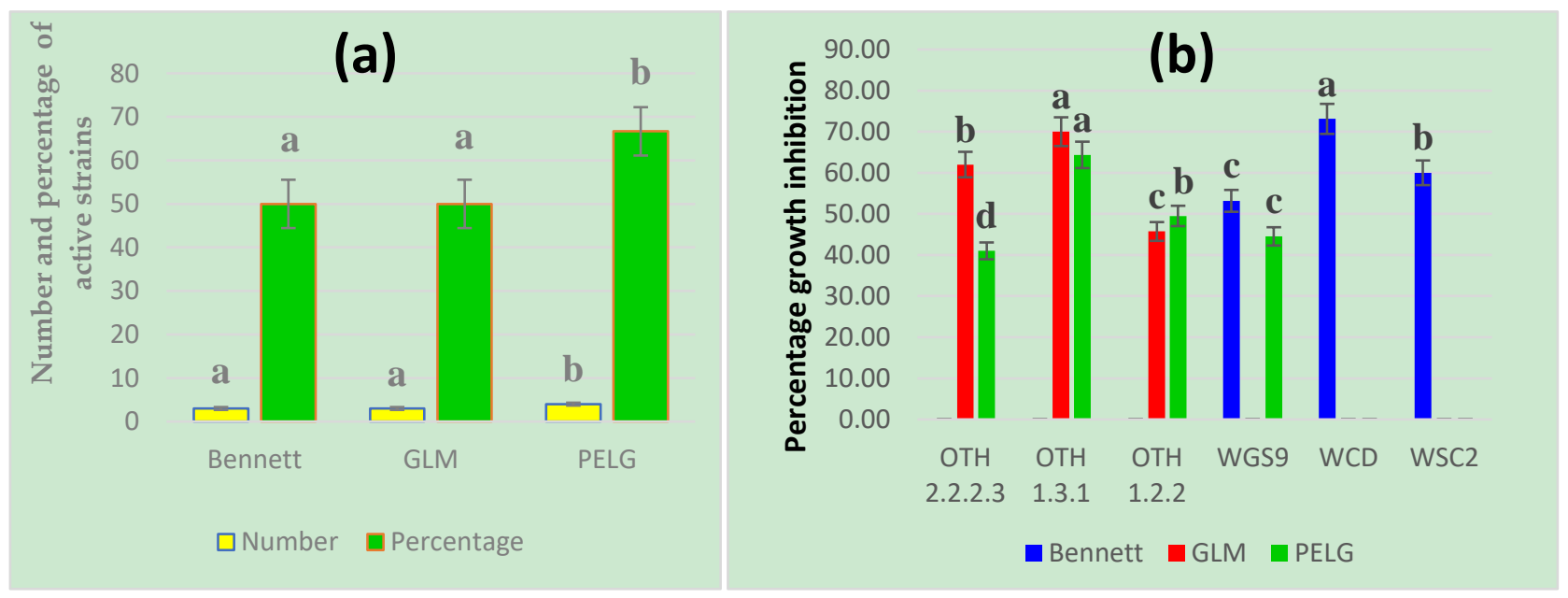

Figure 1. Antifungal activity of actinomycete strains. (a) Number and percentage of active strains; (b) percentage growth inhibition. Results followed by same letters are not significantly different according to Tukey's HSD post-hoc test $(p>0.05)$. 
The antifungal activity against Aspergillus niger on solid media was revealed by the presence of a transparent zone around the actinobacteria agar cylinders. The numbers and percentage of active strains and the percentage growth inhibition are shown in figure 1. Strain antifungal activity was different based on culture medium. On PELG medium, four strains $(67 \%)$ showed antifungal activity. On Bennett medium, three strains (50\%) showed antifungal activity, and the maximum Percentage Growth Inhibition was observed for strain WCD $(73 \%, p<0.05)$. On GLM medium, three strains showed antifungal activity (50\%). In this medium, strain OTH 1.3.1 showed the highest percentage growth inhibition $(70 \%, p<0.05)$. The lowest percentage growth inhibition was recorded for the OTH 2.2.2.3 strain $(41 \%, p<0.05)$.

\subsection{Inoculation of PGPR and Salt-Stress Treatment}

The present study showed that Durum wheat (Triticum durum Desf.) can tolerate $\mathrm{NaCl}$ stress more efficiently when inoculated with thermophilic actinomycete strains as compared with controls. The stimulation of plant morpho-physiological parameters was different according to the inoculated strain and $\mathrm{NaCl}$ concentration which showed variable effects on chlorphyll $\mathrm{a}$, chlorophyll $\mathrm{b}$, total chlorophyll contents (figure 3), proline content (figure 5) and shoots and roots lengths (figure 4).

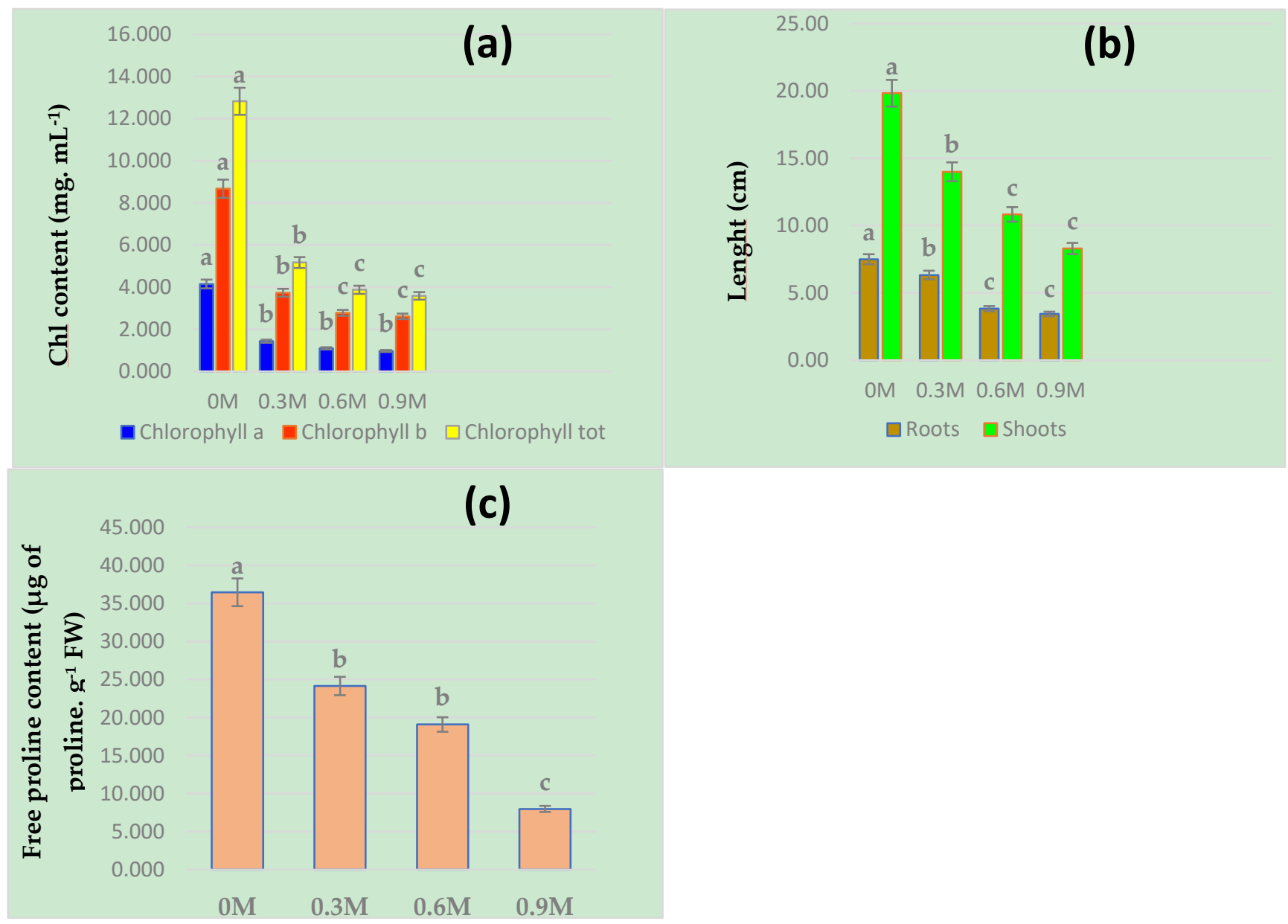

Figure 2. morpho-physiological parameters of Durum wheat uninoculated with the actinomycete strains. (a) contents of chlorophyll a, chlorophyll b and chlorophyll tot; (b) Shoot and root lenght; (c) Free Proline Content. Results followed by the same case letter are not significantly different according to Tukey's HSD post-hoc test $(p>0.05)$ 

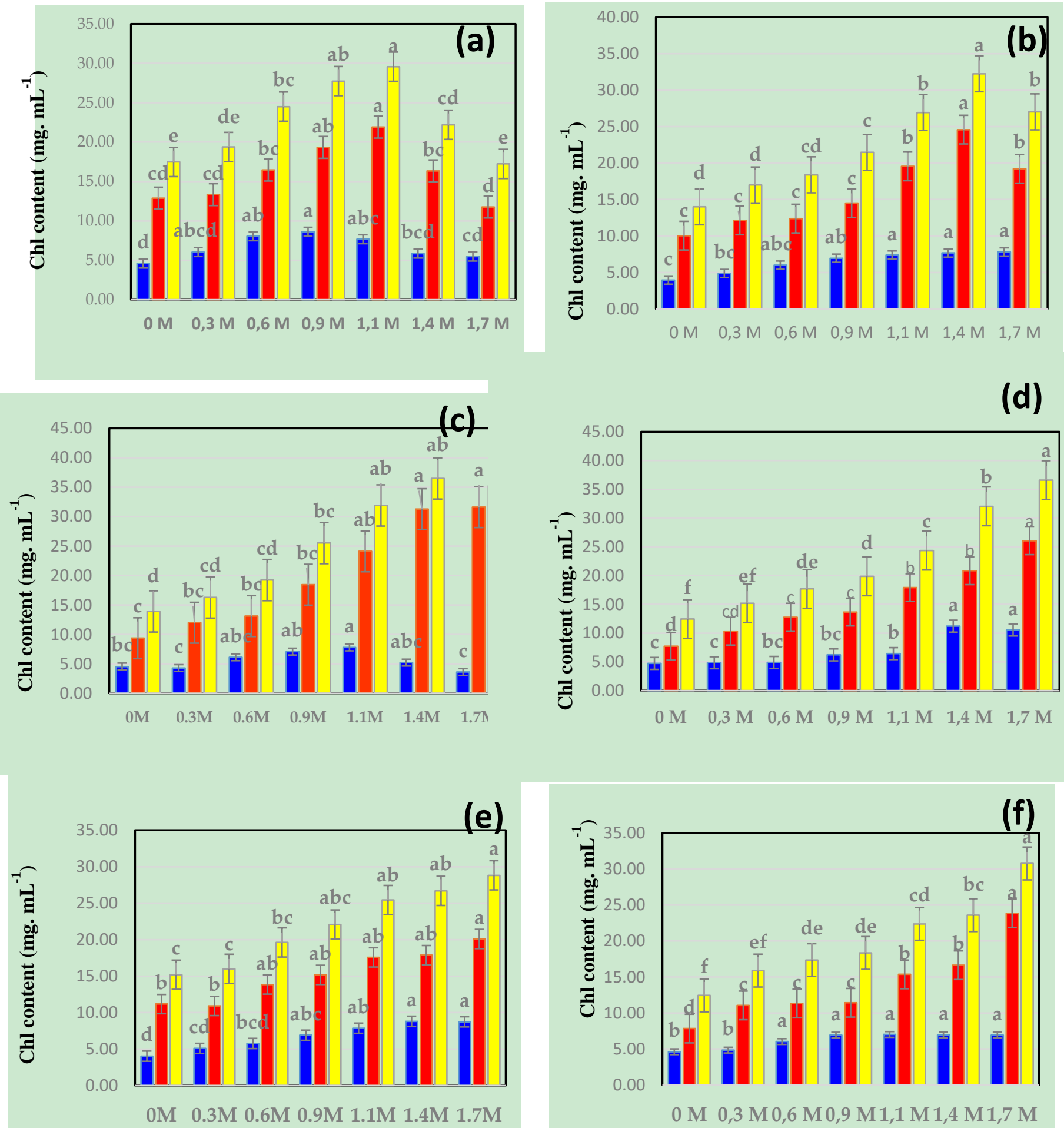

Figure 3. Contents of chlorophyll a (Chla), chlorophyll b (Chlb), and total chlorophyll (Chltot) on Durum wheat inoculated with the selected actinomycete strains: (a) WSC2; (b) WGS9; (c) WCD; (d) OTH 2.2.2.3; (e) OTH 1.3.1; (f) OTH 1.2.2. The results followed by the same letter are not significantly different according to Tukey's HSD post-hoc test $(p>0.05)$. 


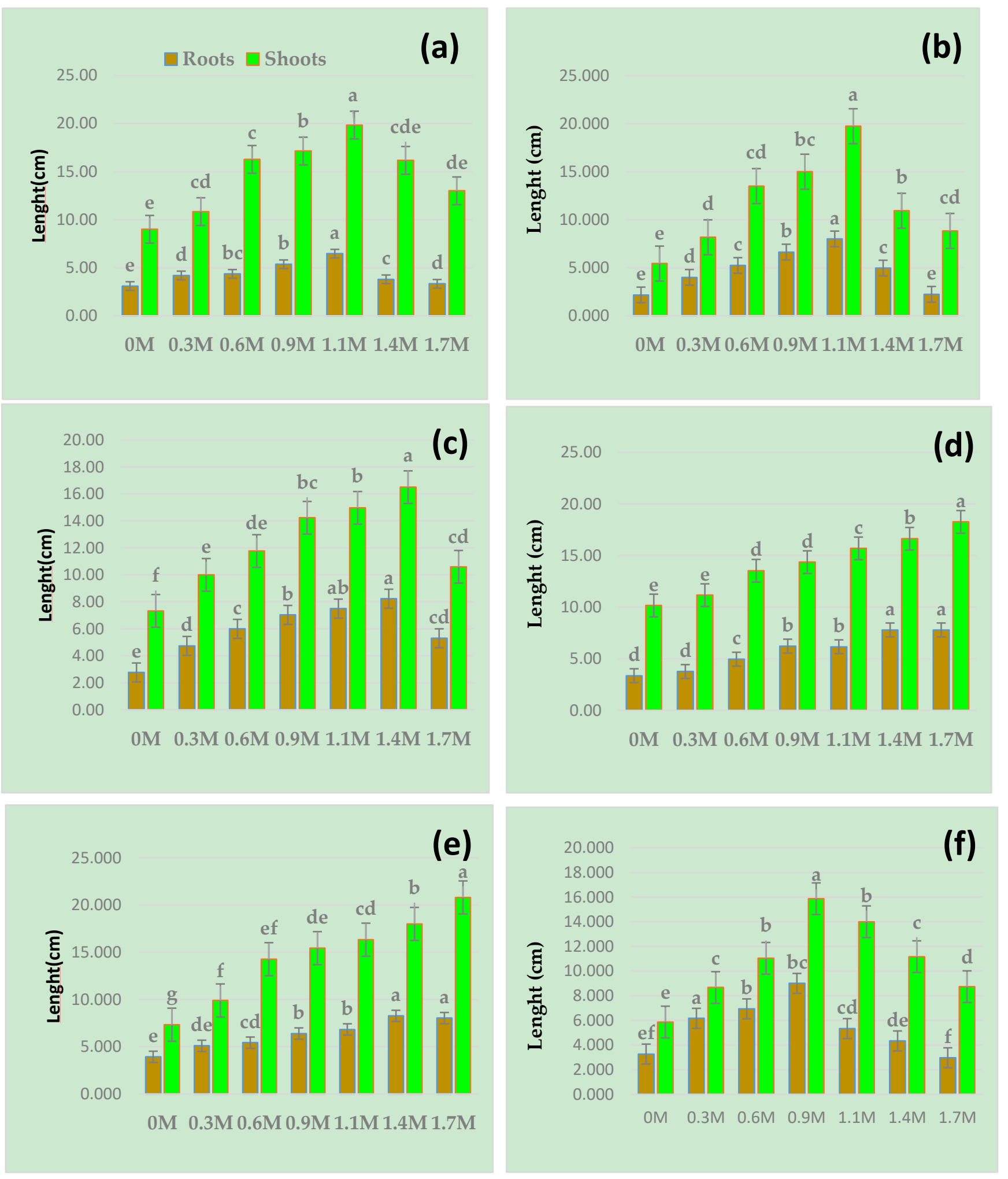

Figure 4. Shoot and root lengths of Durum wheat inoculated with the selected actinomycete strains: (a) WSC2; (b) WGS9; (c) WCD; (d) OTH 2.2.2.3; (e) OTH 1.3.1; (f) OTH 1.2.2. Results followed by the same case letter are not significantly different according to Tukey's HSD post-hoc test $(p>0.05)$. 

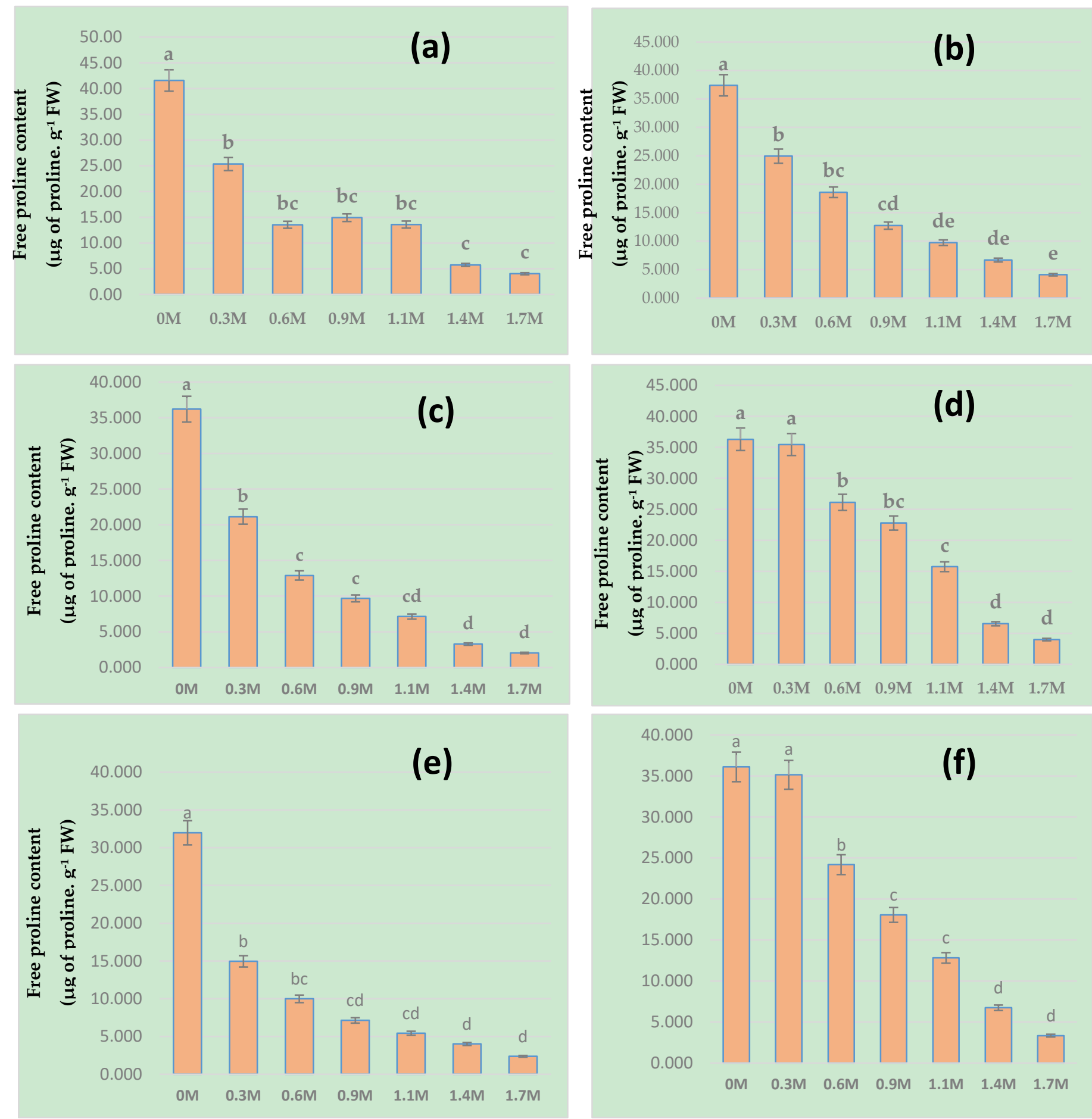

Figure 5. Free proline content of Durum wheat inoculated with the selected actinomycete strains. (a) WSC2; (b) WGS9; (c) WCD; (d) OTH 2.2.2.3; (e) OTH 1.3.1; (f) OTH 1.2.2. Results followed by the same case letter are not significantly different according to Tukey's HSD post-hoc test $(p>0.05)$.

\subsection{Shoot and Root Lenghts}

When $\mathrm{NaCl}$ was added, the shoot and root lengths were affected severely in the case of uninoculated plants $(1,1 \mathrm{M}, 1,4 \mathrm{M}$ and 1,7 M), whereas growth was less reduced in inoculated plants. In the presence of OTH 1.3.1 and WSC2, the growth was significantly better compared to that with other strains. The highest shoot lenghts were recorded for plants inoculated with WSC2, WGS9 and OTH 1.3.1 strains $(p<0.05)$. For root lenghts, plants inoculated with the strain OTH 1.3.1 recorded 
the highest values $(22 \mathrm{~cm}, p<0.05)$. The increase of root and shoot lenghts with respect to the control was $100 \%$ from $1,1 \mathrm{M}$ to $1,7 \mathrm{M}$ of $\mathrm{NaCl}$ solution $(p<0.05)$.

\subsection{Chlorophyll Contents}

Inoculation with actinomycete strains showed variable effects on chlorophyll a (Chla), chlorophyll b (Chlb), and total chlorophyll (Chltot) contents (figure 3). Inoculation with actinomycete strains significantly improved shoot chlorophyll contents of inoculated plants with respect to the uninoculated plants $(p<0.05)$. The highest contents of Chla were observed in plants inoculated with strains OTH 2.2.2.3 (11,21 mg. $\left.\mathrm{mL}^{-1}, p>0.05\right)$. The best contents of Chlb were observed in plants inoculated with strains OTH 2.2.2.3 (36,60 mg. $\left.\mathrm{mL}^{-1}, p>0.05\right)$ at 1,4 $\mathrm{M}$ of $\mathrm{NaCl}$ solution and WCD (36,48 mg. $\mathrm{mL}^{-1}, p>0.05$ ) at $1,7 \mathrm{M}$ of $\mathrm{NaCl}$ solution. Total chlorophyll content increased over the uninoculated control with all the strains. It was highest in the plants treated with the strains WGS9 and OTH 2.2.2.3.

\subsection{Free proline Content}

In Figure 5, the proline stimulation in plants by the thermophilic actinobacteria strains is shown. PGPB-treated plants showed high proline content above $0,9 \mathrm{NaCl}$ stress compared to uninoculated plants. Variable production rates of proline were also detected; the highest content was observed for strain WSC2 (41,58 $\mu \mathrm{g}$ of proline. $\mathrm{g}^{-1} \mathrm{FW}$ Shoots, $\left.p<0.05\right)$, followed by the WGS9 $(37,40 \mu \mathrm{g}$ of proline. $\mathrm{g}^{-1} \mathrm{FW}$ of Shoots, $\left.p<0.05\right)$; OTH 2.2.2.3 (36,31 $\mu$ g of proline. $\mathrm{g}^{-1} \mathrm{FW}$ of Shoots, $\left.p<0.05\right)$; WCD $(36,20$ $\mu \mathrm{g}$ of proline. $\mathrm{g}^{-1} \mathrm{FW}$ of Shoots, $\left.p<0.05\right)$; OTH 1.2.2 (36,1 $\mu \mathrm{g}$ of proline. $\mathrm{g}^{-1} \mathrm{FW}$ of Shoots, $\left.p<0.05\right)$.

\section{Discussion}

The result of the screening carried out on six thermophilic actinomycetes isolated from the saharian soils (Biskra) and water (Ouargla) of south east Algeria show that all the strains present an antifungal activity. The most intense antifungal activity was obtained with Bennett's and GLM media (Figure 2.b). The reduction of the diameters of the inhibition zones of the antifungal activity observed on the strain WSC2 cultivated on the GLM medium as well as the total absence of an antifungal activity of strains OTH 2.2.2.3, OTH 1.3.1 and OTH 1.2.1 when cultivated on a Bennett's medium, confirm the results obtained by Boudemagh et al. [10] and Theobald et al. [11], who showed the influence of the medium composition on the production of antifungal molecules.

Several researchers have already reported similar antifungal activity of actinomycetes isolated from saharan soil of South Algeria, against fungal pathogens. Boudemagh et al [numéro) obtained 27 actinomycete strains from three sahahran soils (south east Algeria), of which, 7,4 \% exhibited antifungal activity against fungi. In a similar study by Lamari et al. [12], three new molecules with antifungal activity were extracted by a species Saccharothrix sp., SA 233, isolated from the Saharian soil of Adrar in south Algeria. According to previous research, ergosterol present in fungal cell membrane has a very high affinity towards polyene antibiotics. Polyene drugs form complexes with ergosterol, forming channels in the fungal membrane that cause leakage of critical intracellular constituents and subsequent cell death [13].

Our results suggest that thermophilic actinomycete strains isolated from saharan sites of Southeast Algeria promote better growth of plants under salt treatment. Plants inoculated with WSC2, WGS9, OTH 1 .3.1, and OTH 2.2.2.3 showed good growth and higher salt tolerance than plants inoculated with other strains. The shoot and root lengths were significantly decreased in uninoculated plants under salt stress, whereas in the presence of PGPR their lengths increased significantly. According to previous research [14], the greater root length of plants is probably due to the availability of higher auxin concentrations.

In this study, PGPR-treated plants showed high proline content under 1,1 M, 1,4M, and 1,7 M $\mathrm{NaCl}$ stress compared to uninoculated plants. Proline is very important biochemical indicator of salinity tolerance in plants as an osmoprotectant that reduce adverse effects of salinity by regulating redox potentials, scavenging oxygen radicals, reducing the acidity in the cell and acts as storage 
compound and nitrogen source [15]. Inoculation with actinomycete strains significantly improved chlorophyll contents of inoculated plants. IAA production plays an important role in growth promotion. This hormone is recognized to stimulate cell elongation with consequent promotion of plant development and chlorophyll content [7].

\section{Conclusion}

From the findings of the present study, it is concluded that the thermophilic actinobacteria tested in the present study can play a pivotal role in conferring salt tolerance in Durum wheat (Triticum durum Desf.) and a good in vitro antifungal control against the inhibitory effects of Aspergillus niger through its plant growth promoting activity.

However, this has to be tested with many other crops to confirm the ability of these strains to confer salt tolerance under field conditions. Therefore, it is plausible to mention that thermophilic actinobacteria are an effective approach for improving the growth of salt-sensitive plants and this strategy could be applied for sustainable agriculture.

Conflicts of Interest: The authors declare no conflict of interest.

\section{Reference}

[1] Riadh, K. ; Wided, M.; Hans-Werner, K.; Chedly, A. Responses of halophytes to environmental stresses with special emphasis to salinity. Adv Bot Res. 2010, 5 (3) : 117-145.

[2] Kitouni, M. ; Boudemagh, A. ; Oulmi, L. ; Reghioua, S. ; Boughachiche, F. ; Zerizer H et al. Isolation of actinomycetes producing bioactive substances from water, soil and tree bark samples of the northeast of Algeria. Journal de Mycologie Médicale. 2005, 15 : 45-51.

[3] Keikha, K. ; Ayatollahi Mousavi, S. A. ; Nakhaei, A. R. ; Yadegari, M. H. ; Shahidi Bonjar, G. H. ;

Amiri, S. In Vitro Evaluation of Enzymatic and Antifungal Activities of Soil-Actinomycetes Isolates and Their Molecular Identification by PCR. Jundishapur J Microbiol, 2015, 8(5) : e14874.

[4] Qi, D. ; Zou, L. ; Zhou, D. ; Chen, Y. ; Gao, Z. ; Feng, R et al. Taxonomy and Broad-Spectrum Antifungal Activity of Streptomyces sp. SCA3-4 Isolated From Rhizosphere Soil of Opuntia stricta. Front. Microbiol. 2019, 10:1390.

[5] Singh, C. ; Singh Parmar, R. ; Jadon, P. ; Bhadauriya, G. ; Kumar, A. ANTIFUNGAL ACTIVITY OF ACTINOMYCETES AGAINST PHYTOPATHOGENS OF GLYCINE MAX.OF CHAMBAL REGION (M.P.) INDIA. International Journal Of Higher Education and Research. 2016, 6 (2) : 122 - 131.

[6] Shahidi Bonjar, G. H. ; Farrokhi, P. R. ; Aghighi, S. ; Shahidi Bonjar, L. ; Aghelizadeh, A. Antifungal Characterization of Actinomycetes Isolated from Kerman, Iran and Their Future Prospects in Biological Control Strategies in Greenhouse and Fild Conditions. Plant Pathology Journal. 2005, 4 (1) : 78-84.

[7] R. Djebaili, M. Pellegrini, M. Smati, M. Del Gallo, Mahmoud Kitouni. Actinomycete Strains Isolated from Saline Soils : Plant-Growth-Promoting Traits and Inoculation Effects on Solanum lycopersicum. Sustainability. 2020, 12, 4617.

[8] Bates, L. ; Waldren, R. ; Teare, I. Rapid Determination of Free Proline for Water-Stress Studies. Plant and soil. 1973, 39:205-207.

[9] Arnon, D.I. Copper enzymes in isolated chloroplasts. Polyphenoloxidase in Beta vulgaris. Plant

Physiol. 1949, 24, 1-15. 
[10] Boudemagh, A. ; Kitouni, M. ; Boughachiche, F. ; Hamdiken, H. ; Oulmi, L. ; Reghioua, S et al. Isolation and molecular identification of actinomycete microflora, of some saharian soils of south east Algeria (Biskra, EL-Oued and Ourgla) study of antifungal activity of isolated strains. Journal de Mycologie Médicale. 2005, 15 : 39-44.

[11] Theobald, U.; Schimana J.; Fiedler HP. Microbial growth and production kinetics of Streptomyces antibioticus TÜ 6040. Antonie Van Leeuwenhoek; 2000, 78:307-13.

[12] Lamari, L. ; Zitouni, A. ; Boudjella, H. ; Badji, B. ; Sabaou, N. ; Lebrihi A et al. New dithiolopyrrolone antibiotics from Saccharothrix sp. SA 233. I. Taxonomy, fermentation, isolation and biological activities. J Antibiot. 2002, 55:696- 701.

[13] Nafis, A. ; Elhidar, N. ; Oubaha, B. ; Samri, S. ; Niedermeyer, T. ; Ouhdouch, Y. Screening for Non-polyenic Antifungal Produced by Actinobacteria from Moroccan Habitats : Assessment of Antimycin A19 Production by Streptomyces albidoflavus AS25. IJMCM. 2018, 7 (2).

[14] Shukla, P. S. ; Agarwal, P. K. ; Jha, B. Improved Salinity Tolerance of Arachis hypogaea (L.) by the Interaction of Halotolerant Plant-Growth-Promoting Rhizobacteria. J Plant Growth Regul. DOI 10.1007/s00344-011-9231-y.

[15] Tavakoli, M. ; Poustini, K. ; Alizadeh, H. Proline Accumulation and Related Genes in Wheat Leaves under Salinity Stress. J. Agr. Sci. Tech. 2016, Vol. 18: 707-716.

Publisher's Note: MDPI stays neutral with regard to jurisdictional claims in published maps and institutional affiliations.

(C) 2020 by the authors. Submitted for possible open access publication under the terms and conditions of the Creative Commons Attribution (CC BY) license (http://creativecommons.org/licenses/by/4.0/). 